\title{
Market architecture for TSO-DSO interaction in the context of European regulation
}

\author{
Andrei Z. Morch \\ Hanne Sæle \\ SINTEF Energy Research \\ Trondheim, Norway
}

\author{
Dario Siface \\ Gianluigi Migliavacca \\ RSE S.p.A \\ Milano, Italy
}

\author{
Helena Gerard \\ VITO NV \\ Mol, Belgium
}

\author{
Ivana Kockar \\ University of Strathclyde \\ Glasgow, UK
}

\begin{abstract}
Following the overall European political goals, massive efforts were recently made to promote an accelerated integration of renewable energy sources (RES) in Europe, creating several operational challenges. One of the key approaches to resolve these is to help harness RESs in an efficient and cost-effective way is to utilise flexibility which can be provided by Distributed Energy Resources (DERs) which include active demand participation, energy storage and electric vehicles. The present paper is based on results and learnings of H2020 project SmartNet (2016-2019), where five coordination schemes for TSO-DSO interaction, necessary for procurement and activation of ancillary services were developed and comparatively evaluated. The paper discusses how different coordination schemes all have specific benefits and attention points related to operation of the TSO and DSO grids, other market participants involved and the market operation in general.
\end{abstract}

Index Terms - market architecture, transmission and distribution, European regulation

\section{INTRODUCTION}

Setting the 2020 climate and energy targets in 2007 was an important milestone, indicating a paradigm shift for the European power industry, which used to be one of the most conservative sectors. Massive efforts were made to promote an accelerated integration of renewable energy sources (RES) in Europe, however, the intermittent nature of these resources may require higher levels of ancillary services (AS). In addition, considerable share of RESs are connected at the distribution systems and which means that they change the nature of these networks that are becoming active, with possible changes in directions of power flows. One of the key approaches to help harness RESs in an efficient and costeffective way is to utilise flexibility which can be provided by Distributed Energy Resources (DERs) which include active demand participation, energy storage and electric vehicles. Some of the main aspects of the transition towards low-carbon energy systems envisioned by new European regulation and roadmaps [1] include market based provision of ancillary services by DERs that need to be give a level playing field to participate in all electricity/energy and ancillary services markets, at both transmission and distribution networks.
DERs can be used as a valuable resource for frequency control, voltage regulation and congestion management, both at transmission and distribution levels. This will however require a significant modification of the present interaction between TSOs and DSOs and corresponding changes in the today's roles and responsibilities. In addition, operation of systems with high levels of DERs as well as design and operation of associated energy and ancillary services markets will need new tools and underpinning regulation and codes. The aim of this paper is to discuss some of the regulatory aspects that need to be considered and address

The present paper is based on results and learnings of H2020 project SmartNet (2016-2019), where five coordination schemes for TSO-DSO interaction, necessary for procurement and activation of ancillary services were developed. These different schemes span from the situation of a complete centralized control over AS market to the creation of different local markets run by DSOs and one AS market run by the TSO. Comparative assessment of these schemes supports the initial idea and allows to compare these, based on results of cost-benefit analysis (CBAs).

\section{SMARTNET COORDINATION SCHEMES}

The project has developed five alternative architectures or coordination schemes (CSs) that each present a different way of organizing the coordination between transmission and distribution system operators (TSOs and DSOs), when distributed resources (production, storage or demand) are used for ancillary services (for details, see [2] and [3]). Each coordination scheme is characterized by a specific set of roles and responsibilities, taken up by system operators and a detailed market design.

- Centralized AS market model (CS_A), where the TSO operates a market for both resources connected at transmission and distribution level, without involvement of the DSO. TSO contracts services directly from DER. No congestion management is carried out for distribution grids;

- Local AS market model (CS B), where the DSO organizes a local market for resources connected at the DSO-grid and, after solved local grid constraints, 
offers the remaining to the AS market managed by TSO (procuring balancing and congestion management).

- Shared balancing responsibility model (CS_C), where balancing responsibilities are divided between TSO and DSO according to a predefined schedule. The DSO organizes a local congestion and balancing market using local DER, while the TSO has no access to resources connected at the distribution grid.

- Common TSO-DSO AS market model (CS D), where the TSO and the DSO have a common objective to decrease costs to satisfy both the need for resources by the TSO and the DSO. This common objective could be realized by the joint operation of a common market (centralized variant) or the dynamic integration of a local market, operated by the DSO, and a central market, operated by the TSO (decentralized variant).

- Integrated flexibility market model (CS_E), where the market is open for both regulated and nonregulated market parties, which requires the introduction of an independent market operator to guarantee neutrality. TSOs, DSOs and commercial market parties contract DER in a common flexibility market.

Table I provides a short overview of different benefits and attention points for the suggested coordination schemes.

In order to compare CS performance, SmartNet has developed a simulation platform, modelling in detail T\&D networks, ancillary services markets and implementing a very detailed dataset of generators and loads. Simulations are carried out on midterm scenarios (time horizon 2030) for Spain, Denmark and Italy to identify the best TSO-DSO coordination scheme for each country.

The same platform is also implemented in a laboratory in order to test real network equipment on the developed simulation scenarios (hardware-in-the-loop). The simulation results were further assessed by using a set of specific key performance indicators (KPIs). The overall method and outcomes of the evaluation are presented in detail in [5].

TABLE I SUMMARY OF THE BENEFITS AND ATTENTION POINTS FOR SMARTNET COORDINATION SCHEMES. SOURCE [4]

\begin{tabular}{|c|c|c|}
\hline $\begin{array}{l}\text { Coordination } \\
\text { Scheme }\end{array}$ & Benefits & Attention points \\
\hline $\begin{array}{l}\text { Centralized AS } \\
\text { market model }\end{array}$ & $\begin{array}{l}\text { Efficient scheme in case when TSO is the only buyer } \\
\text { for the service. Having only one market is low in } \\
\text { operational costs and supports standardized processes. } \\
\text { The most in line with current regulatory framework }\end{array}$ & No real involvement of DSO.DSO grid constraints not always respected \\
\hline $\begin{array}{l}\text { Shared balancing } \\
\text { responsibility model }\end{array}$ & $\begin{array}{l}\text { The TSO will need to procure a lower amount of AS. } \\
\text { Local markets might create lower entry barriers for } \\
\text { small scaled DERs. }\end{array}$ & $\begin{array}{l}\text { Total amount of AS to be procured by TSO and DSO maybe higher in this } \\
\text { scheme. BRPs might face higher costs for balancing. Small local markets } \\
\text { may not be liquid enough to provide sufficient resources for the DSO }\end{array}$ \\
\hline $\begin{array}{l}\text { Common TSO-DSO } \\
\text { AS market model }\end{array}$ & $\begin{array}{l}\text { Total cost of AS for TSO and DSO are minimized. TSO } \\
\text { and DSO make optimal use of each other. }\end{array}$ & $\begin{array}{l}\text { Individual cost of TSO and DSO might be higher compared to other } \\
\text { schemes. Allocation of costs between TSO and DSO could be difficult. }\end{array}$ \\
\hline $\begin{array}{l}\text { Integrated flexibility } \\
\text { market model }\end{array}$ & $\begin{array}{l}\text { Increased possibilities for BRPs to solve imbalances in } \\
\text { their portfolio. High liquidity and relative low prices } \\
\text { due to large number of buyers and sellers. }\end{array}$ & $\begin{array}{l}\text { Independent market operator needed to operate the grid. Negative impact } \\
\text { on the development and liquidity of intraday markets. TSO and DSO need } \\
\text { to share data with Independent Market Operator (IMO). }\end{array}$ \\
\hline
\end{tabular}

\section{EVAluAtion OF THE SMARTNet COORDinAtion SCHEMES (CSS)}

In order to identify compliance of the proposed coordination schemes with the current regulation and overall position of the main stakeholders it has been identified 25 main issues, here referred to as topics of interest, which are associated with solutions and assumptions, which are essential for the min outcomes of the project. These topics have been evaluated in a comprehensive screening study, based on more than 40 different documents such as position papers, strategies, roadmaps and legislation/regulation (EU Directives, Network guidelines, national regulatory Decisions) (see [4] for the complete overview). The following lines present the assessment of the coordination schemes that has been considered by the SmartNet project.

First, the Cost Benefit Analysis (CBA) results [6], based on the numerical simulations performed on the three national 2030 scenarios (Italy, Denmark and Spain), are briefly introduced. Then a list of remarks mainly but not only related to regulation is showed [7].

From the analysis of the proposed TSO-DSO coordination schemes, it is evident how the amount of services requested by the DSO (i.e. congestion management) is making the difference in terms of cost effectiveness. In fact, looking at 
the Italian case (Figure 1.a) where a consistent amount of congestions is expected to occur at distribution level [8], market architectures including distribution network constraints are more optimal than the ones featured by coordination schemes in which the DSO cannot buy flexibility (CS_A). This is particularly noticeable by looking at the amount of activated aFRR, indicating the residual imbalance after the market clearing (resulting from forecasting error and unforeseen congestions).
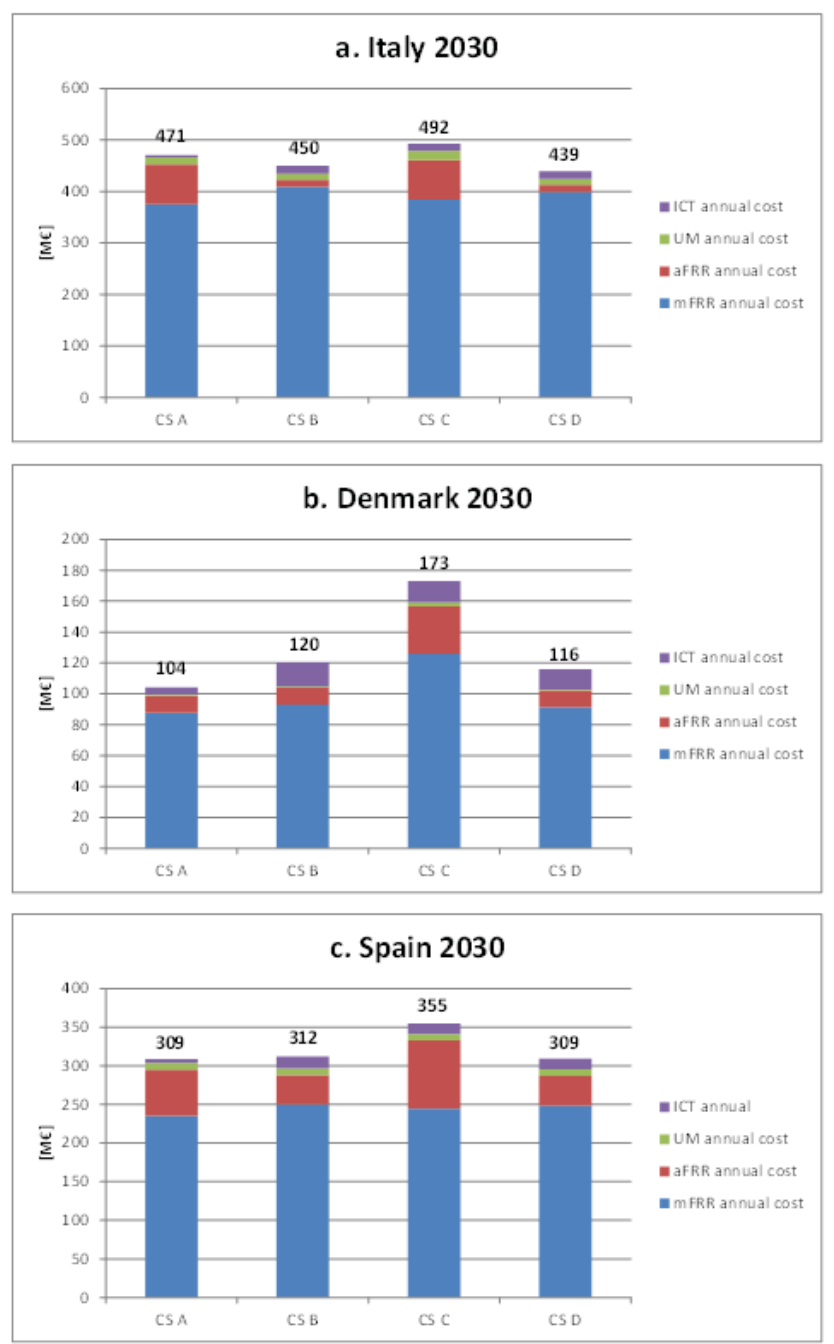

Figure 1. Results of the CBA analysis of the three National 2030 Scenario simulated

The simulated 2030 scenario for Denmark, instead, is returning opposite results (Figure 1.b). The negligible amount of congestions at distribution level, as well as the low amount of reserve needed for the balancing services, is making the cost of ICT decisive in determining the most beneficial coordination scheme. The middle ground is obtained for the Spanish scenario (Figure 1.c), where the cost of ICT equals the benefits achieved thanks to the inclusion of distribution constraints in market clearing algorithms.

All the considered scenarios are affected by scarcity and illiquidity of resources at distribution level specifically for the provision of local balancing services (this scarcity also affect CS_B which has slightly lower performance than CS_D). This assumption is making the application of CS_C quite inefficient, having the market clearing algorithms often unable to satisfy the balancing requirements and resulting in high operation (mFRR $+\mathrm{aFRR})$ costs. More details on the simulation environment and the cost figures, including the one assigned to information and communication technology (ICT), are detailed in [5], [6], [9], [10].

\section{A. Centralized AS market model (CS_A)}

CS_A defines that DSOs involvement is limited to prequalification of resources at distribution level to guarantee that no congestions are generated by the activation of such resources. Thus, the DSOs must have a full control over their network, and for this purpose appropriate investments in ICT are needed. So, DSOs should be made able to compare such investments in ICT with the ones on the Distribution Network (DN) expansion, within a long-term planning perspective and in coordination with Transmission, since the look has to be broadened on the efficiency of the whole system. Regulation should thus consider this need, for example by passing from the present CAPEX approach to the TOTEX one.

\section{B. Local AS market model (CS_B)}

CS B defines that a local congestion market is run for the DN. After DSOs activate the needed resources to solve local congestions, the remaining ones are made available to TSOs for the global balancing market, that takes place in a second step.

This two-step procedure may likely reduce the economic efficiency of the system. For instance, when the knowledge of the whole System status is available (e.g. as like as what happens in CS_D, discussed above), the imbalance caused by the solution of a local congestion could help to solve a possible opposite imbalance, reducing the amount of resources activated. If the local market is run before the global balancing one, not only the above operation is impossible, but, also, the local. System is rebalanced after congestion is solved, and in the second step furthermore activations are needed to solve the global imbalance. Furthermore, since local networks may be very small and with a very limited amount of resources available, the risk of lack of liquidity and/or exercise of market power increases.

Obviously, these risks are related to all the possible market schemes (not only the four schemes assessed within the SmartNet project), but they increase if the perimeter of the market is reduced. Thus, the solution is to enlarge as much as possible the perimeter of the market by allowing the small DSOs to join up in a single, and wider, local market; by increasing the amount of resources participating to the local market, for example introducing market products tailored on their technical needs, particularly on the demand side.

Local and global markets may also have different timing. In this case, they have to be properly coordinated in order to avoid double acceptance of bids presented on both markets (e.g. a common database of resources shared between TSO and DSOs without time correlation so that once a resource 
has been selected by one operator it becomes unavailable for the others, as in the "Common Sequenced Market" in [5]).

\section{Shared balancing responsibility model (CS_C)}

CS_C is set up with two separated markets for Transmission and Distribution, with a fixed exchange profile imposed at the boundary between the two; DSOs are given also balancing responsibilities on local networks, on which, then, they must have a complete control: further investments in ICT are fundamental.

The firm constraint imagined in SmartNet at the boundary between T\&D is very strong and may result in a large loss of optimality (if not even prevent to find a solution, with the need to activate Unwanted Measures). To add some degrees of flexibility at this boundary could be beneficial to the efficiency of such a scheme, but it is difficult to figure out how to maintain the complete separation between the two market. However, this complete separation may also have some good effects, as, for example, to prevent high (local) prices to spread along the whole System. On the other hand, these high prices may be consequence of the separation, that likely prevents the activation of the more efficient resources if they are "on the wrong side"; furthermore, also compensation of local and global imbalances with different signs is always impeded.

Again, liquidity and/or market power exercise risk may likely be significant here, also because they are related not only to congestion management but also for balancing. For all these reasons, CS_C is expected to perform poorly by an economic point of view; this is confirmed by simulations, as shown in Figure 1. Since this is the only scheme that implement a local balancing market, we can ascribe a share of that inefficiency also to this peculiarity, so that we can infer that balancing should be coped with globally (as diffusely acknowledged - see for instance [11]).

\section{Common TSO-DSO AS market model (CS D)}

Finally, CS_D is characterized by a common AS Market for both DSOs and TSOs. This market includes all technical and network constraints, so that a consistent computational effort for its clearing is needed; on the other hand, since all the information is shared and available, the very optimal solution can be found and we can expect high economic performances, as confirmed by our simulations (Figure 1). Obviously, this purpose needs a strong TSO-DSO cooperation; in particular, regulation should take care of all the data sharing implications.

A relevant drawback of such a scheme (it affects all the schemes, but this one in particular since no other significant drawbacks are present) is the impact of forecasting error: it may drive to activate resources for solving forecasted imbalances and/or congestions that in real time do not occur, so that counteractions are needed in real time (as it happened in some simulations). Pushing gate closure as close as possible to real time and/or increasing market clearing frequency could help in reduce this impact; however, some technical constraints may not be easily eliminated: as already said, the computational effort to clear the common market with all the network constraints included.
In any case, the experience of the three Pilot projects in SmartNet (in particular of the Spanish Pilot) showed clearly that the use of flexibility resources gives to DSOs more options, than the simple network reconfiguration, to solve local network issues, leading to an evident increase in the economic and technical efficiency of the local network operation.

\section{E. Integrated flexibility market model (CS_E)}

CS E considers the case of a common market in which both regulated (TSO and DSO) and Commercial Market Parties (CMPs) procure flexibilities. It may reveal promising to eliminate different markets gate closure overlapping, since it could be considered a "mix" of Intraday and AS Markets, and effective for certain kind of AS (for instance, for solving balancing issues). However, it may have some significant drawbacks, as:

- $\quad$ since CMPs can change their output in this market, uncertainty may arise both about the real amount of congestion and imbalance in the System and, then, about how many resources are needed by TSOs and DSOs;

- $\quad$ since TSOs and DSOs compete in equal terms with other CMPs for flexibility, in some scenarios they could no longer be sure even to acquire the resources they need;

- the high level of competition could result in very high market prices.

Due to the need to consider game theory elements, this scheme is characterized by a high level of mathematical complexity, so, within the SmartNet Project, it was neither investigated as deeply as the other ones nor simulated. Thus, it remains field for further investigations

\section{CONCLUSIONS}

The different coordination schemes all have specific benefits and attention points related to operation of the TSO and DSO grids, other market participants involved and the market operation in general. The choice of the appropriate coordination scheme is dependent on multiple factors such as the type of ancillary service, normal operation versus emergency situations, the state of the grid, the amount of RES installed, the current market design and the regulatory framework. Moreover, the choice for a specific coordination scheme does not imply that this scheme could never be adapted.

Across coordination schemes, there is a gradual increase of the role and responsibilities of the DSO. Dependent on the national evolution, a country can evolve from one coordination scheme to another. In particular, the Centralized AS market model (CS_A), the Common TSO-DSO AS market model (CS_D) (centralized variant) and the Integrated flexibility (CS E) market model share a common market architecture in terms of market platform and ICT requirements. A shift between these coordination schemes is mainly a question of a change in roles and responsibilities. The Shared balancing responsibility model could be seen as a duplication of the same market architecture as well. Also, the 
Local AS market model and the Common TSO-DSO AS market model (decentralized variant) share a common market architecture.

The feasibility of the implementation of each coordination scheme is very dependent upon the regulatory framework. The Centralized AS market model is the most in line with current regulations. The other coordination schemes would require considerable changes with respect to roles and responsibilities of TSOs and DSOs.

The implementation of a coordination scheme is also influenced by the national organization of TSOs and DSOs, e.g. the number of system operators (both TSOs and DSOs) and the way they currently interact.

In addition, the implementation of certain coordination schemes will have an impact on other markets, such as the Intraday markets. Dependent on the services offered in the AS market, and compared to the Intraday markets (IDM), these markets might be able to co-exist or alternatively, may need to be integrated. Although TSO-DSO coordination could be organized on a country level, it is important to integrate national TSO-DSO coordination set-ups within the process of EU harmonization and integration.

\section{ACKNOWLEDGMENT}

The authors gratefully acknowledge the financial support from the European Union's Horizon 2020 research and innovation programme under grant agreement No 691405 to project SmartNet and from Research Council of Norway and user partners of FME CINELDI - Centre for intelligent electricity distribution, an 8 year Research Centre under the FME-scheme (Centre for Environment-friendly Energy Research, 257626/E20).

\section{REFERENCES}

[1] European Commission, "Proposal for regulation of the European Parliament and of the Council on the internal market for electricity (recast)", 2017.

[2] H. Gerard, E. Rivero and D. Six, "Basic schemes for TSO-DSO coordination and ancillary services provision", SmartNet Project Tech. Rep. D1.3. 2016

[3] H. Gerard, E. Rivero, D. Six, "Coordination between transmission and distribution system operators in the electricity sector: A conceptual framework". Utilities Policy 50, 40-48. https://doi.org/10.1016/j.jup.2017.09.011

[4] A. Morch et al, "Evaluation on project results related to a number of models and roadmaps", SmartNet Project, Tech. Rep. D6.2. 2019

[5] C. Madina, I. Riaño S. Gómez et al. "Cost-Benefit Analysis of TSO-DSO coordination to operate flexibility markets". CIRED 2019 - 25th International Conference and Exibition on Electricity Distribution. Paper 1632. June 2019

[6] I. Gómez et al., "Cost-benefit analysis of the selected national cases”, SmartNet Project, Tech. Rep. D4.3. 2019

[7] D. Siface et al., "Policy recommendations to implement and/or overcome barriers and enable TSO-ISO integration", SmartNet Project. Tech. Rep. D6.3. 2019

[8] H. Svendsen, M. Rossi et al., "Scenario set up and simulation results," SmartNet Project, Tech. Rep. D4.2. 2019

[9] M. Rossi et al., "Testing TSO-DSO Interaction Schemes for the Participation of Distribution Energy Resources in the Balancing Market: the SmartNet Simulator", CIRED 2019 - 25th International Conference and Exhibition on Electricity Distribution, June 2019

[10] P. Kuusela, P. Koponen, H. Xu, I. Kockar, "An ICT cost comparison of different market structures for distributed ancillary services," CIRED 2019 - 25th International Conference and Exibition on Electricity Distribution, June 2019

[11] European Commission, "Proposal for a Directive of the European Parliament and of the Council on common rules for the internal market in electricity", 2302 2017. [Online]. Available: https://eurlex.europa.eu/legalcontent/EN/TXT/?uri=CELEX\%3A52016PC0864R\%2801\%29 